\title{
A caracterização da gestão da rede de atenção à saúde bucal no estado do Rio de Janeiro pelo perfil regional
}

\author{
I ${ }^{1}$ Marcos Alex Mendes da Silva, ${ }^{2}$ Eduardo Tavares Lima Trajano, \\ ${ }^{3}$ Renata Ferraiolo Gueiros, ${ }^{4}$ Amanda de Almeida Jannuzzi Mendes I
}

Resumo: A Rede de Atenção à Saúde Bucal (RASB) é constituída por três elementos: a população, a estrutura e o modelo de atenção à saúde. As açóes dessa rede são distribuídas pelos pontos de atenção primário, secundário e terciário. O objetivo do texto consiste em conhecer o seu estágio de desenvolvimento na regiáo serrana do estado do Rio de Janeiro e as maiores fragilidades. Constitui um estudo de casos, exploratório e transversal, com amostra intencional e aplicação de questionário estruturado. A interpretação dos dados foi realizada por meio da média dos pontos e pela análise de correlaçáo de Pearson, que demonstrou o predomínio da RASB incipiente $\left(\sum=\right.$ 137 pontos) e a correlação forte das variáveis: adoção de protocolos clínicos X capacidade resolutiva da atençáo secundária, manutenção dos equipamentos odontológicos X existência de laboratórios de apoio, existência de ações educativas $X$ frequência de sua utilização, frequência de sua utilização X açóes reabilitadoras para o câncer bucal, as açóes reabilitadoras para o câncer bucal X existência de um plano de cuidado $(r \geq 0,90)$. Concluiu-se que a RASB encontra-se em estágio incipiente e as fragilidades envolvem dificuldades na adscrição, nos encaminhamentos, na emissão de laudos radiológicos, na adoção de prontuário eletrônico e na apropriação dos instrumentos de gestão.

> Palavras-chave: Gestão em Saúde, Regionalização, Saúde Bucal.

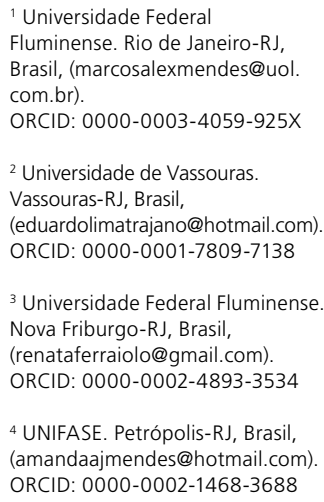

${ }^{1}$ Universidade Federal

Fluminense. Rio de Janeiro-RJ,

Brasil, (marcosalexmendes@uol. com.br).

ORCID: 0000-0003-4059-925X

2 Universidade de Vassouras.

Vassouras-RJ, Brasil,

(eduardolimatrajano@hotmail.com). ORCID: 0000-0001-7809-7138

${ }^{3}$ Universidade Federal Fluminense. Nova Friburgo-RJ, Brasil, (renataferraiolo@gmail.com). ORCID: 0000-0002-4893-3534

${ }^{4}$ UNIFASE. Petrópolis-RJ, Brasil, (amandaajmendes@hotmail.com). ORCID: 0000-0002-1468-3688

Recebido em: 25/10/2019 Aprovado em: 25/05/2020 Revisado em: 05/10/2021 


\section{Introdução}

Já no final da década de 1980, a constituição brasileira propunha um novo sistema de saúde, baseado nos princípios da universalidade do acesso, da integralidade da atençáo à saúde e da equidade das necessidades da populaçáo, todos ancorados e sustentados pela solidariedade e pela incorporação dos determinantes sociais na produção do cuidado em saúde. Essa nova proposta materializou-se na implantação do Sistema Único de Saúde (SUS), composto inicialmente por uma rede hierarquizada e regionalizada de unidades de saúde, distribuídas por diferentes níveis de complexidade da atenção.

A partir de 2011, o SUS assume uma proposta de funcionamento em rede integrada de açôes para superar as dificuldades impostas à regionalização e garantir acesso aos serviços de saúde, com o sistema orientado por um conjunto coordenado de pontos de atenção, no intuito de prestar assistência contínua e integral, em substituição ao modelo hierarquizado e piramidal até então dominante. Os desafios enfrentados por esta mudança não foram poucos, considerando que os mesmos níveis de atenção organizados agora seriam reproduzidos de forma horizontal e ainda, em cada rede temática estabelecida (MENDES, 2011).

Segundo Basso et. al. (2019), as Redes de Atenção à Saúde (RAS) são constituídas por três elementos fundamentais: a população e as regiōes de saúde, a estrutura operacional e o modelo de atenção à saúde. Neste modelo, a concepção de hierarquia é substituída pela poliarquia e o sistema organiza-se de forma horizontal, sem ordem e grau de importância entre eles, apenas se diferenciando pelas respectivas densidades tecnológicas que os caracterizam. Neste sentido, rompe-se com a gestão baseada exclusivamente na oferta, característica dos sistemas fragmentados e, institui-se a gestão baseada nas necessidades de saúde da população, elemento essencial das RAS.

Dois estudos descritivos caracterizam a rede de atenção à saúde bucal em diferentes contextos: no primeiro, realizado no Estado de Santa Catarina em 2014, foi possível identificar a presença dos elementos constitutivos anteriormente descritos: População, Estrutura operacional e Modelo de atenção, assim como o nível de integração entre eles. Por meio de questionário estruturado autoaplicável e de dados secundários, chegou-se ao resultado que mostra que alguns municípios atuavam de forma bastante desarticulada; enquanto outros ofereciam serviços de saúde bucal organizados, de modo a seguir os pressupostos da rede regionalizada, com a atenção básica pautada 
pela Estratégia Saúde da Família (ESF) e com possibilidades de coordenar a atenção à saúde (GODOI, MELLO e CAETANO, 2014). No segundo estudo, realizado em alguns municípios da regiáo serrana do Rio de Janeiro em 2015, com o intuito de se analisar o perfil e o processo de trabalho de cirurgióes dentistas inseridos na Estratégia Saúde da Família (ESF), os autores concluíram que a inclusão da equipe de saúde bucal, que priorizou a prevenção e promoção de saúde bucal na ESF, ampliou o acesso da população à assistência odontológica. Por outro lado, alguns cirurgiōes dentistas encontraram dificuldades em distinguir e atuar na necessidade epidemiológica que essas pessoas apresentavam. Observou-se que é necessário portanto, estimular o trabalho desses profissionais em equipe multiprofissional e promover melhorias nas condiçôes de trabalho (MORAES, KLIGERMAN e COHEN, 2015).

De forma complementar aos estudos supracitados, Pinto et al. (2016) destacaram que a lógica das RAS mostrou-se favorecida pelo desenho da Política Nacional de Saúde Bucal, que se estrutura em eixos estratégicos que abrangem a vigilância em saúde, a expansão da oferta de serviços na atenção primária até a assistência na média e na alta complexidade, além da transversalidade com outros programas ministeriais.

Portanto, a atenção à saúde bucal no SUS acompanhou a lógica das RAS e suas ações foram distribuídas pelos pontos de atenção primária à saúde (APS), representados pelas unidades de saúde da família e pelas unidades básicas de saúde; pelos pontos de atenção secundária, nos Centros de Especialidades Odontológicas (CEO) do Brasil Sorridente e pelos pontos de atenção terciária, com procedimentos realizados no âmbito hospitalar e de alta densidade tecnológica. Apesar desse avanço da organização em rede da saúde bucal no SUS, o processo de gestão ainda carece de tomada de decisóes qualificada, por inexistir diagnósticos situacionais baseados em indicadores do estágio de evolução dessa rede assistencial. Existem iniciativas isoladas de avaliação e monitoramento da saúde bucal na APS, assim como, indicadores do funcionamento dos $\mathrm{CEO}$, porém, inexistem informaçóes que avaliem o estágio de desenvolvimento de toda a rede assistencial em saúde bucal (atenção primária, secundária e terciária) e, ainda, que considere a percepção do gestor no gerenciamento dessa integração.

Para responder a essas inquietaçóes com vistas ao melhor funcionamento do campo de saúde bucal como rede articulada, definiu-se como objetivo desse estudo, conhecer o estágio de desenvolvimento da RASB na região serrana do estado do Rio de Janeiro e as maiores fragilidades. 


\section{Materiais e métodos}

O estudo realizado foi do tipo estudo de casos, exploratório, transversal, desenvolvido na regiáo serrana do estado do Rio de Janeiro, composta por dezesseis municípios e uma população estimada em 873.837 habitantes, subdivididos em três microrregiōes: Serrana I (1 município), Serrana II (5 municípios) e Serrana III (10 municípios). A amostra inicial era composta por 12 coordenadores municipais de saúde bucal (CSB), abrangendo todas as microregiôes, já que 4 municípios não possuíam esse cargo regulamentado e não atendiam a esse critério de inclusão. Assim 12 CSB que responderam ao Instrumento de Diagnóstico do Estágio de Desenvolvimento da Rede de Atenção à Saúde (MENDES, 2011), adaptado para a versão saúde bucal e validado em estudo anterior (LEAL e al, 2014). A opção pela realização do estudo em apenas uma região de saúde do estado ocorreu em função do pioneirismo da aplicação do instrumento, que, embora validado, ainda carecia de uma análise crítica. Foi feito estudo piloto com 5 professores de odontologia em Saúde Coletiva da Universidade Federal Fluminense que, ao responder o questionário, apontaram um número excessivo de perguntas e algumas com certa complexidade no entendimento, exigindo um longo tempo para respondê-lo.

Para não descaracterizá-lo, optou-se por aplicá-lo presencialmente pelos pesquisadores, no sentido de minimizar vieses de informaçáo, com possibilidade de esclarecimentos durante a sua aplicação. O instrumento proposto é composto por 107 perguntas, distribuídas por 7 domínios, tais como, as características da população, da atenção primária à saúde local, dos pontos de atenção à saúde secundários e terciários, dos sistemas de apoio (diferentes laboratórios, exames complementares, assistência farmacêutica), dos sistemais logísticos (ferramentas de apoio logístico), do sistema de governança da rede (instrumentos de gestão) e do modelo de atenção à saúde predominante (Leal e tal., 2014).

Os dados foram primeiramente analisados descritivamente no programa Biostat5, por meio da soma dos pontos alcançados pelo questionário aplicado e classificados conforme os parâmetros propostos para o instrumento: de 0 a 80 pontos - RASB fragmentada; de 81 a 160 pontos - RASB incipiente; de 161 a 240 pontos - RASB avançada; e de 241 a 321 pontos - RASB integrada.

A segunda etapa de interpretação dos dados foi feita pela análise de correlação de Pearson das variáveis do estudo, no sentido de se verificar quais correlaçôes eram 
mais fortes e estatisticamente mais significativas, para ajudar a compreender melhor os problemas que impediam a conformação de uma rede integrada e avançada de atenção à saúde bucal. O coeficiente de correlação de Pearson (r) varia de -1 a 1 e quanto mais próximo de 1, independente do sinal, maior é o grau de dependência estatística linear entre elas. Para a interpretação dos dados a partir do valor de r, foram estabelecidos os seguintes critérios: $\mathrm{r}=0$ a 0,5 (correlação fraca); $\mathrm{r}=0,6$ a 0,7 (correlação moderada) e r = 0,8 a 0,9 (correlação forte) (HAIR, 2009).

$\mathrm{O}$ protocolo de pesquisa recebeu parecer favorável à sua execução pelo Comitê de Ética e Pesquisa do Instituto de Saúde de Nova Friburgo da Universidade Federal Fluminense, sob o número CAAE 60619116.2.0000.5626. Todos os entrevistados assinaram o termo de consentimento livre e esclarecido, sendo respeitados os princípios da Resolução 466, de 12 de dezembro de 2012.

\section{Resultados e discussão}

A tabela 1 mostra o perfil dos municípios analisados por meio da classificaçáo inicial obtida pela pontuação do instrumento de coleta de dados, com predomínio da RASB incipiente na região serrana $\left(\sum\right.$ dos pontos $=137$ ), o que demonstra que os próprios gestores reconhecem a dificuldade enfrentada na integração dos pontos de atenção à saúde bucal. E que, mesmo os municípios que possuem uma rede avançada, com fluxo de usuários entre os níveis de atenção à saúde satisfatório, o maior valor ( $\sum$ dos pontos $=203$ ) ainda está distante do valor máximo do instrumento para essa faixa $\left(\sum\right.$ dos pontos $=240-$ RASB avançada). Nenhum município foi classificado no estágio máximo de desenvolvimento da rede ( $\Sigma$ dos pontos entre 240 a 321), enquanto dois alcançaram o menor estágio (municípios $G$ e J, respectivamente, $\Sigma$ dos pontos 69 e 84), dimensionando a RASB ainda fragmentada nestes municípios, cujo serviço de saúde bucal local encontra-se totalmente desorganizado e com pouca resolutividade no fluxo dos usuários. Alguns vieses podem ter influenciado essa caracterização, sobretudo, o pouco conhecimento do gestor sobre a sua própria rede ou a sua ocupaçáo recente no cargo de gestão. 
Tabela 1. Perfil da Rede de Atenção à Saúde Bucal (RASB) na Regiáo Serrana do Estado do Rio de Janeiro

\begin{tabular}{llll}
\hline Municípios & Média por município & S dos pontos & Classificação (RASB) \\
\hline A & $1,3 \pm 0,9$ & 154 & RASB incipiente \\
\hline B & $1,0 \pm 1,0$ & 130 & RASB incipiente \\
\hline $\mathrm{C}$ & $1,3 \pm 1,0$ & 160 & RASB incipiente \\
\hline $\mathrm{D}$ & $0,9 \pm 0,9$ & 100 & RASB incipiente \\
\hline $\mathrm{E}$ & $1,8 \pm 0,9$ & 159 & RASB incipiente \\
\hline $\mathrm{F}$ & $2,2 \pm 1,1$ & 203 & RASB avançada \\
\hline $\mathrm{G}$ & $0,3 \pm 0,8$ & 69 & RASB Fragmentada \\
\hline $\mathrm{H}$ & $1,0 \pm 0,9$ & 128 & RASB incipiente \\
\hline $\mathrm{I}$ & $1,6 \pm 1,2$ & 199 & RASB avançada \\
\hline $\mathrm{J}$ & $0,6 \pm 0,7$ & 84 & RASB Fragmentada \\
\hline $\mathrm{L}$ & $1,2 \pm 1,0$ & 146 & RASB incipiente \\
\hline $\mathrm{M}$ & $1,0 \pm 0,9$ & 120 & RASB incipiente \\
\hline & Média de todos os municípios & 137 & RASB incipiente \\
\hline
\end{tabular}

Os dados dos 12 municípios foram expressos na forma de soma e média \pm desvio padrão. A classificação das RASB foi feita de acordo com a soma dos pontos obtidos (Leal et al., 2014)

Quando analisados os motivos desta desarticulação, as maiores dificuldades enfrentadas na integração dessa rede assistencial referem-se à ausência de elementos estruturantes importantes, tais como, os critérios claros na definição das populações adscritas na regionalização de suas áreas de trabalho, sejam eles de risco epidemiológico ou por idade; a referência formal com garantia de realização de próteses dentárias, assim como para ortodontia e implantodontia e para todo o nível terciário, sem que os serviços estivessem preparados para absorver urgências e emergências em hospitais, sobretudo, 24 horas; a emissão de laudos que acompanhassem os exames radiológicos, pela falta de profissional qualificado; a adoção de prontuário eletrônico individual e/ou familiar; e, por fim, a utilização da central de regulação de vagas para os serviços de odontologia. Todas essas variáveis apresentaram mais da metade das respostas $(n \geq 6)$ com grau 0 , ou seja, sem que o respondente soubesse do que se tratava a pergunta. 
A tabela 2, sobre os coeficientes de correlação, mostra o perfil da RASB por domínio, com apresentação exclusiva das variáveis com forte correlação entre elas (valor de $r \geq 0,75$ ), com influência direta sobre o estágio de desenvolvimento da RASB. Dentre estas, todas as correlações selecionadas foram positivas, ou seja, com crescimento diretamente proporcional entre as variáveis escolhidas. Os maiores valores de $\mathrm{r}$ encontravam-se nos domínios $\mathrm{C}$ (Os pontos de atenção à saúde secundária e terciária), D (Sistema de apoio) e G (Modelos de atenção à saúde), todos com algum valor $\geq 0,90$.

Tabela 2. Perfil da Rede de Atenção à Saúde Bucal (RASB) da Região Serrana por domínio

\begin{tabular}{|c|c|c|}
\hline Variáveis & Correlaçáo de Pearson (r) & Coeficiente de determinaçáa $(\mathrm{r} 2)$ \\
\hline \multicolumn{3}{|c|}{ DOMÍNIO A - População } \\
\hline $10 \mathrm{vs.} 12$ & 0,77 & $77 \%$ \\
\hline \multicolumn{3}{|c|}{ DOMÍNIO B - Atenção primária a saúde (APS) } \\
\hline 17 vs. 21 & 0,85 & $85 \%$ \\
\hline
\end{tabular}

DOMÍNIO C - Os pontos de atenção à saúde secundária e terciária

44 vs.45 $0,90 \%$

DOMINIO D - Os sistemas de apoio

\begin{tabular}{lll}
\hline 51 vs.52 & 0,87 & $87 \%$ \\
\hline 53 vs.54 & 0,94 & $94 \%$ \\
\hline 62 vs.64 & 0,78 & $78 \%$ \\
\hline
\end{tabular}

DOMÍNIO E - Os sistemas logísticos

\begin{tabular}{lll}
\hline $\mathbf{6 8}$ vs. 69 & 0,82 & $82 \%$ \\
\hline 70 vs. 75 & 0,81 & $81 \%$ \\
\hline 75 vs. 76 & 0,80 & $80 \%$ \\
\hline
\end{tabular}

DOMÍNIO G - Modelos de atenção à saúde

\begin{tabular}{lll}
\hline $\mathbf{9 0}$ vs.92 & 0,80 & $80 \%$ \\
\hline $\mathbf{9 1}$ vs.92 & 0,90 & $90 \%$ \\
\hline $\mathbf{9 1}$ vs.93 & 0,82 & $82 \%$ \\
\hline $\mathbf{9 1}$ vs.96 & 0,76 & $76 \%$ \\
\hline
\end{tabular}

continua... 


\begin{tabular}{|c|c|c|}
\hline Variáveis & Correlação de Pearson (r) & Coeficiente de determinaçáo (r2) \\
\hline 92 vs.93 & 0,93 & $93 \%$ \\
\hline 92 vs.96 & 0,84 & $84 \%$ \\
\hline 92 vs. 113 & 0,77 & $77 \%$ \\
\hline 93 vs.94 & 0,81 & $81 \%$ \\
\hline 93 vs.94 & 0,81 & $81 \%$ \\
\hline 93 vs.96 & 0,92 & $92 \%$ \\
\hline 93 vs. 113 & 0,75 & $75 \%$ \\
\hline 94 vs.95 & 0,86 & $86 \%$ \\
\hline 94 vs.96 & 0,82 & $82 \%$ \\
\hline 94 vs. 114 & 0,82 & $82 \%$ \\
\hline 95 vs.96 & 0,76 & $76 \%$ \\
\hline 96 vs.97 & 0,83 & $83 \%$ \\
\hline 96 vs.99 & 0,79 & $79 \%$ \\
\hline 96 vs. 114 & 0,79 & $79 \%$ \\
\hline 98 vs.99 & 0,79 & $79 \%$ \\
\hline 100 vs.101 & 0,81 & $81 \%$ \\
\hline 102 vs. 106 & 0,89 & $89 \%$ \\
\hline 103 vs. 105 & 0,95 & $95 \%$ \\
\hline 103 vs. 106 & 0,85 & $85 \%$ \\
\hline 104 vs. 105 & 0,90 & $90 \%$ \\
\hline 104 vs.106 & 0,76 & $76 \%$ \\
\hline 110 vs. 111 & 0,84 & $84 \%$ \\
\hline
\end{tabular}

Os dados dos 12 municípios foram expressos através do valor de $\mathrm{r}$ e $\mathrm{r} 2$. Foram considerados somente valores de $r>0,75$.

No domínio C, que aborda os pontos de atenção à saúde secundária e terciária, verifica-se que a adoção de protocolos clínicos relativos às intervençóes mais relevantes está diretamente relacionada com a capacidade da atenção secundária em realizar todos os procedimentos da sua competência $(r=0,90)$, demonstrando a importância dos protocolos clínicos no encaminhamento adequado dos usuários 
para níveis com maior potencial de resolução. O que já parecia óbvio, confirmou-se pelo valor de $\mathrm{r}$, ou seja, os protocolos clínicos bem delineados definem melhor os encaminhamentos, que quando adequados, possuem alto potencial de resolução no nível para o qual o usuário foi encaminhado.

Moysés (2009) destacou duas razóes para adoção dos protocolos em odontologia. Primeiro, para padronizar condutas que contribuam com a organização do cuidado, envolvendo, a definição de território, a cobertura, a porta de entrada, os resultados sanitários, entre outros e em segundo, para orientar a prática clínica assistencial do profissional no encaminhamento correto dos usuários, filtrando encaminhamentos desnecessários. Essas razóes basearam-se no Manual de Especialidades em Saúde Bucal, publicado pelo Ministério da Saúde no mesmo período (Brasil, 2008), que visava colaborar com o estabelecimento de critérios de referência e contrarreferência entre a Atenção Primária/ Saúde da Família (APS/SF) e os CEO, assim como instrumentalizar a prática clínica e de gestão relativas às principais especialidades odontológicas, aperfeiçoando a dinâmica da rede de serviços do SUS. Apresentava ainda, um conjunto de princípios e recomendaçóes elaborados para facilitar a tomada de decisão apropriada na atenção aos pacientes, em situaçóes específicas, dotando cada serviço de um método ordenado nas diferentes especialidades.

Souza et al. (2015) concluíram em seu estudo sobre fluxo de usuários, que o indivíduo ao ser encaminhado ao $\mathrm{CEO}$ pelo dentista da Atenção Básica possui maior chance de retornar a este nível de atenção, sem a resolução do problema, se não possuir uma referência formal que lhe garanta o atendimento no nível secundário. Assim, a forma de encaminhamento torna-se fator decisivo e a referência dada pelo dentista da UBS contribui para a integralidade na atenção em saúde bucal, na medida em que essa referência seja baseada em protocolos clínicos pertinentes. Por outro lado, as outras formas de encaminhamento e demanda livre podem comprometer o desempenho dos CEO e a sua capacidade de realizar os procedimentos da sua competência, quando os mesmos são utilizados para atendimentos de baixa complexidade. Apesar da fragilidade observada no sistema de referência e contra referência entre a Atenção Básica e o CEO, ressalta-se a possibilidade de reorganização do modelo de porta de entrada no sistema, com novas estratégias de fluxo, embora ainda predomine em muitas realidades essa inversão assistencial e/ou a ausência de adoção de protocolos eficazes. 
No domínio $\mathrm{D}$, que envolve os sistemas de apoio, pôde-se observar que a manutenção dos equipamentos odontológicos possui correlação forte e positiva com a existência de laboratórios de apoio para os serviços prestados ( $\mathrm{r}=0.94)$, sobretudo de prótese dentária, que se encontra alocado nas unidades básicas de saúde e é o mais utilizado recurso de apoio logístico. Novamente o valor de $\mathrm{r}$ confirma a força da relação, na qual o bom funcionamento do equipamento odontológico envolve os laboratórios de prótese, tendo sido essa uma fragilidade apontada pela maioria dos municípios que enfrentam dificuldades na oferta das próteses e não conseguem consorciar essa ação no nível regional, por diferentes motivos, inclusive pela estrutura física inadequada

Donabedian (1988) já afirmara há tempos atrás que a qualidade assistencial estava relacionada com a tríade: estrutura, processo e resultados, e que destes, a estrutura adequada favorecia a oferta de açóes integrais, assim como Amorim et. al. (2013) relacionaram o aspecto físico dos estabelecimentos assistenciais à sua funcionalidade. Arquitetura, engenharia, organização, administração e manutenção são também meios para viabilizar a proteção à saúde da população, e não somente os processos, tal como, os gestores expuseram neste domínio.

Obviamente, as dificuldadesenfrentadas não decorrem exclusivamenteda estrutura física dos cenários assistenciais, mas eles representam uma parcela significativa dos motivos que contribuem para essas dificuldades, embora, para minimizar o problema, o Ministério da Saúde tenha fomentado ações de reestruturação de unidades básicas de saúde, por meio do Programa Requalifica UBS (Brasil, 2017) e, especificamente na saúde bucal, do próprio Programa Brasil Sorridente (Brasil, 2004), ambos com incentivo financeiro para pretendida readequação.

E no domínio G, que caracteriza os modelos de atenção à saúde, destacam-se algumas correlaçóes fortes, tais como, entre a existência de açóes educativas sobre os fatores de risco para afecçôes bucais e a frequência de sua utilização, entre a frequência de utilização de açóes educativas e as açôes reabilitadoras para o câncer bucal, entre as açóes reabilitadoras e a existência de um plano de cuidado para enfrentamento dos fatores de risco $(r=0,90 ; r=0,94 ; r=0,90$, respectivamente) e, por fim, entre os contratos de gestão e as auditorias clínicas $(\mathrm{r}=0,93)$, que embora estivessem no mesmo domínio, necessitam de reflexão diferenciada.

As ações educativas sobre fatores de risco, sua frequência e as ações reabilitadoras oferecidas aos portadores de câncer bucal estão fortemente associadas e diretamente 
ligadas ao modelo de atenção à saúde adotado, considerando que as RAS investem no cuidado integral e na vigilância em saúde e a forte correlação entre as variáveis confirma essa assertiva. Porém, um dos problemas da maioria dos modelos assistenciais hegemônicos decorre do foco exclusivo na doença, mesmo quando se oferece um programa com uma lógica de antecipação dos agravos, as propostas ainda se mantêm voltadas, prioritariamente, para a recuperação da saúde (Mendes, 2011). O modelo Biomédico de explicação do processo saúde doença, embora ultrapassado, ainda predomina e orienta a atenção à saúde atualmente.

No presente estudo, essa fragilidade do modelo assistencial se reproduz, pois ao aumentar as açōes educativas, aumenta-se também a sua frequência $(\mathrm{r}=0,90)$, porém ao aumentar a sua frequência, deveriam se diminuir as ações reabilitadoras (valor de $\mathrm{r}$ negativo), o que náo acontece, quando analisada exclusivamente a correlação linear proposta pelo valor de $\mathrm{r}(\mathrm{r}=0,94)$. Para explicar esse fenômeno, torna-se necessário uma investigação mais detalhada das razões que motivaram as respostas dos coordenadores sobre a abordagem do paciente com risco de afecçôes bucais, entretanto, supõe-se que as açôes educativas não estejam sendo capazes de "frear" a quantidade de procedimentos reabilitadores em decorrência do câncer bucal, ou, na pior hipótese, que elas não estejam modificando hábitos e/ou estilos de vida, já que são frequentemente adotadas, conforme as respostas.

O modelo assistencial que preconiza a odontologia na Estratégia Saúde da Família (ESF) busca a promoção de saúde e a prevenção dos agravos, porém, não desconsidera a etapa recuperadora, que juntas, definem a integralidade do cuidado em saúde bucal, exemplificado pelas variáveis associadas deste domínio. O programa Brasil Sorridente (Brasil, 2004) pavimenta esse caminho, com espaços reservados à educação, ao diagnóstico precoce e ao tratamento do câncer de boca no SUS, desde as USF, passando pelo CEO até chegar ao ambiente hospitalar, todos com a supervisão do cirurgião-dentista. Porém, na região serrana, a dificuldade encontra-se nos níveis mais complexos da abordagem dos pacientes diagnosticados com câncer bucal, cujo tratamento ocorre exclusivamente na capital do estado, sem que nenhum dos 12 municípios possua referência para esse tratamento no nível reabilitador, mesmo aqueles que são sede das microrregióes.

Outro aspecto relacionado pela correlação das variáveis desse domínio envolve a existência de instrumentos de gestáo consolidados e os mecanismos de monitoramento e avaliação instituídos, e embora haja compromisso gestor com tais iniciativas, a 
maioria encontra-se no âmbito central das secretarias de saúde e não sob domínio do coordenador de saúde bucal (CSB). A associação entre as variáveis demonstra a importância da relação, com 50\% dos relatos dos CSB afirmando funcionamento adequado, tanto dos instrumentos de gestão, quanto dos mecanismos de avaliação e monitoramento, porém, com outros $50 \%$ reforçando a ausência do conhecimento do gestor sobre tais mecanismos.

O decreto n. 7508/2011, a Lei Complementar n. 141/2012 e a portaria n. 2135/2013 estabelecem as diretrizes para o planejamento em saúde no âmbito do SUS, com macro instrumentos de gestão, que devem conter as metas e os resultados da Saúde Bucal em cada período planejado e avaliado. O Plano de Saúde contém as ações previstas na Programação Anual de Saúde e representa o elenco de intenções a ser realizado, assim como, o relatório de gestão expressa os resultados alcançados pela execução da referida programação (BRASIL, 2015).

Embora se espere que os CSB conheçam os instrumentos de gestão, em muitas realidades estes instrumentos são de domínio e manuseio exclusivo do setor de planejamento das secretarias de saúde, mesmo que o coordenador tenha sido o responsável pela alimentação de todo sistema de informação do seu setor durante quatro anos. Esse desenho operacional reserva à coordenação de saúde bucal um papel secundário na gestão plena do serviço, confirmado pelas respostas do questionário de $50 \%$ dos gestores que desconhecem os instrumentos de gestão, e pelos $50 \%$ que conhecem, porém não participam de sua elaboração e aplicação.

\section{Considerações finais}

A região serrana do estado do Rio de Janeiro apresenta sua rede de atenção à saúde bucal no estágio incipiente de desenvolvimento, ou seja, carece de recursos e meios que incentivem a integração dos pontos de atenção dentro dos municípios e entre eles, favorecendo a comunicação entre a atenção primária, secundária e terciária, assimo como, a resolutividades dos problemas de saúde bucal. A pouca articulação que existe, não é capaz de garantir a atenção integral à população.

Este tipo de diagnóstico realizado na região serrana permitirá aos pesquisadores e à gestão municipal de saúde bucal de qualquer cenário estudado terem subsídios para reorientar o planejamento das suas açóes, organizando os fluxos dos usuários pelos pontos de atenção nos diferentes níveis de complexidade da saúde bucal no 
SUS, com vistas à diminuição das filas de espera para o tratamento odontológico mais complexo e à satisfação dos pacientes que utilizam essa rede.

Foi possível conhecer as maiores fragilidades da articulação dessa rede assistencial, representadas pela ausência de clareza nos critérios de definição das populaçóes adscritas às áreas de trabalho das equipes de atenção primária; pouca efetivadade no mecanismo de referência formal ente os níveis de atenção; dificuldade na emissão de laudos que acompanhem os exames radiológico; ausência na doção de prontuário eletrônico individual e/ou familiar; e, por fim, a baixa utilização da central de regulação de vagas para os serviços de odontologia.

Observou-se ainda que as ações preventivas e reabilitadoras do câncer de boca ocorrem na mesma intensidade na região de saúde, sem que o primeiro nível de atenção das ações preventivas impactasse o nível mais complexo, o reabilitador, característica de modelos assistenciais ainda centrados na doença, com pouca valorização das medidas educativas e promocionais.

No campo da gestáo, o estudo confirma a necessidade que o CSB tem de qualificar a sua gestão no SUS, apropriando-se dos indicadores, dos instrumentos de gestão e participando efetivamente da elaboração do Plano de Saúde municipal, que absorve todas as demandas loco regionais de saúde bucal.

\section{Referências}

AMORIM, GM.; QUINTÃO, ECV.; MARTELLI-JUNIOR, H.; BONAM, PRF. Prestação de serviços de manutenção predial em estabelcimentos assistenciais de saúde. Ciênc Saúde Col., v. 18 , n. $1,2013$.

BASSO, MB; NUNES, MB; CORREA, LBC; VIEIRA, CN; VILARINHO, JLPS; PUCCA JUNIOR, GA. A construção da rede de atenção à saúde bucal no Distrito Federal, Brasil. Ciênc. saúde coletiva, v.24 n.6 , 2019.

BRASIL. Conselho Nacional de Secretários de Saúde. Gestão do SUS/ Conselho Nacional de Secretários de Saúde. Brasília: CONASS, 2015.

BRASIL. Ministério da saúde secretaria de atenção à saúde departamento de atenção básica coordenação nacional de saúde bucal. Diretrizes da politica nacional de saúde bucal. Brasília, 2004.

BRASIL. Ministério da saúde. Secretaria de atenção à saúde . Portaria $n^{\circ} 381$, de 6 de fevereiro de 2017. Dispóe sobre as transferências, fundo afundo, de recursos financeiros de capital ou corrente, do ministério da saúde a estados, distrito federal e municípios destinados à execução de obras de construção, ampliação e reforma. 
BRASIL. Ministério da Saúde. Secretaria de Atenção à Saúde. Departamento de Atenção Básica. Manual de especialidades em saúde bucal / Ministério da Saúde, Secretaria de Atenção à Saúde, Departamento de Atenção Básica. - Brasília : Ministério da Saúde, 2008.

DONABEDIAN, A. The quality care. How can it be acessed? JAMA 1988:2601743-8

GODOI, H.; MELLO, AL.; CAETANO, JC. Rede de atenção à saúde bucal: organização em municípios de grande porte de Santa Catarina, Brasil. Cad. Saúde Pública. v. 30; n. 2, 2014

HAIR, JF et al.. Multivariate Data Analysis. New Jersey: Prentice-Hall; 2009.

LEAL, DL et al.. Adaptação da versão saúde bucal do instrumento de diagnóstico do estágio de desenvolvimento da rede de atenção à saúde. Cad. Saúde Pública v.30 n.6, 2014.

MENDES, EV. As redes de atenção à saúde. 2a Ed. Brasília: Organização PanAmericana da Saúde; 2011.

MORAES, LB; KLIGERMAN, DC; COHEN, SC. Análise do perfil sociodemográfico e do processo de trabalho do cirurgião-dentista inserido no Programa de Saúde da Família em três municípios da região serrana do Estado do Rio de Janeiro. Physis. vol.25, n.1, 2015

MOYSÉS, SJ. Protocolos de Saúde Bucal. I Encontro de Centros de Especialidades Odontológicas e Equipes de Saúde Bucal na Estratégia Saúde da Família. Curitiba, 2009.

PINTO, MHB.; ARAÚJO, ME.; FUJIMAKI, M.; DITTERICH, RG.; TERADA, RSS.; MARTELLI, PJL. As rede de atenção à saúde bucal - o papel do CEO. IN: MARTELLI, PJL.; FIGUEIREDO, N.; GOES, PSA. Os caminnhos da saúde bucal no Brasil - um olhar quali e quanti sobre os centros de espeicalidades odontológicas (CEO) no Brasil. Recife: UFPE, 2016.

SOUZA, GC.; LOPES, MLDS.; RONCALLI, AG.; MEDEIROS-JUNIOR, A.; CLARACOSTA, IC. Referência e contra referência em saúde bucal: regulação do acesso aos centros de especialidades odontológicas. Rev. salud pública, v. 17, n. 3, 2015. 


\section{Abstract}

\section{The characterization of the management of the oral health care network in the state of Rio de Janeiro by the regional profile}

The Oral Health Care Network (OHCN) consists of three elements: the population, the structure and the model of health care, and its actions distributed by the primary, secondary and tertiary care points. The objective is to know its stage of development in the highland region of the state of Rio de Janeiro and the biggest weaknesses. Exploratory and crosssectional case study with intentional sample and application of structured questionnaire. Data interpretation was performed by means of the points average and Pearson's correlation analysis, which demonstrated the predominance of incipient $\mathrm{OHCN}\left(\sum=137\right.$ points) and the strong correlation of the variables: adoption of clinical protocols vs. secondary care resolution capacity. , maintenance of dental equipment $\mathrm{X}$ existence of support laboratories, existence of educational actions $\mathrm{X}$ frequency of its use, between frequency of its use $\mathrm{X}$ rehabilitation actions for oral cancer, among the rehabilitation actions for oral cancer $\mathrm{X}$ existence of a care plan ( $r \geq 0.90)$. It was concluded that the RASB is in an incipient stage and the weaknesses involve difficulties in the subscription, referrals, issuing radiological reports, the adoption of electronic medical records and the appropriation of management instruments.

> Keywords: Health Management; Regional Health Planning; Oral Health. 